\title{
Measurement of nanopatterned surfaces by real and reciprocal space techniques
}

\author{
P. Siffalovic, K. Vegso, M. Jergel, E. Majkova, J. Keckes ${ }^{1}$, G. A. Maier ${ }^{2}$, M. Cornejo ${ }^{3}$, B. Ziberi ${ }^{3}$, \\ F. Frost ${ }^{3}$, B. Hasse ${ }^{4}$, J. Wiesmann ${ }^{4}$ \\ Institute of Physics SAS, Dubravska cesta 9, 84511 Bratislava, Slovakia \\ ${ }^{1}$ Erich Schmid Institute for Materials Science, Jahnstr. 12, A-8700 Leoben, Austria \\ ${ }^{2}$ Materials Center Leoben Forschung GmbH, Roseggerstr. 12, A-8700 Leoben, Austria \\ ${ }^{3}$ Leibniz-Institut für Oberflächenmodifizierung e. V., 04318 Leipzig, Germany \\ ${ }^{4}$ Incoatec GmbH, Max-Planck-Str. 2, 21502 Geesthacht, Germany
}

A newly developed laboratory grazing-incidence small-angle X-ray scattering GISAXS system capable of statistical measurements of surface morphology at the nanometer scale was developed. The potential of the GISAXS system is compared to the AFM technique for a nanopatterned silicon surface produced by ion-beam erosion. The characteristic period of the ion-beam induced ripples and their lateral correlation length were estimated from AFM. Using GISAXS the reciprocal space map of surface morphology was measured and analyzed. The two microfocus $X$-ray sources emitting radiation at the $\mathrm{Cu}-\mathrm{K}_{\alpha}$ and $\mathrm{Cr}-\mathrm{K}_{\alpha}$ were $u$ sed. The lateral periods of ripples obtained by the reciprocal space mapping techniques match the results of real space techniques. The setup has the potential to monitor and control the deposition process and formation of nanostructures with sufficient temporal and spatial resolution.

Keywords: AFM, GISAXS, nanostructures, ion-beam sputtering

\section{INTRODUCTION}

$\mathrm{N}$ ANOSTRUCTURED surfaces and interfaces represent a challenge for current science and technology. Traditional approaches relying on the "top-down" technologies like nanolithography are expensive, rather slow and limited in terms of projection area. Emerging "bottomup" technologies based on the self-organization can provide a viable solution to certain problems related to modification of interfaces at nanoscale. There is a need to apply a new non-local technique allowing an in-situ monitoring and control of the deposition process leading to formation of nanostructures at surfaces and interfaces. The method should be compatible with standard requirements of the PVD and CVD techniques and fast enough to monitor the evolution of nanostructures during the deposition process. In this contribution we present a novel table-top grazingincidence small-angle X-ray scattering (GISAXS) system suitable for monitoring and control of the ion-beam erosion technique $[1,2]$. The GISAXS results are compared to the real space atomic force microscopy (AFM) technique.

\section{SUBJECT \& METHODS}

The ion-beam induced ripples on the silicon substrate were prepared by an experimental technique described in detail by Ziberi et al. [3]. Prior to inspection the sample was ultrasonically cleaned in chloroform and dried in a stream of nitrogen gas. Finally, the surface of the sample was cleaned in a home-built UV-ozone reactor working with ambient air and low pressure $\mathrm{Hg}$ lamp. The surface morphology was inspected by a commercial AFM device (Dimension Edge, Bruker AXS) employing a tapping imaging mode (cantilever OTESPA, Bruker AXS). The GISAXS system is a homebuilt apparatus based on the microfocus X-ray source equipped with a focusing Montel optics ( $\mathrm{I} \mu \mathrm{S}$, Incoatec). In this study we used two X-ray sources emitting the radiation at $\mathrm{Cu}-\mathrm{K}_{\alpha}(\lambda=0.154 \mathrm{~nm})$ and $\mathrm{Cr}-\mathrm{K}_{\alpha}(\lambda=0.229 \mathrm{~nm})$ lines. The size of the focused X-ray beam at $560 \mathrm{~mm}$ distance from the source was $0.25 \mathrm{~mm}$ in terms of full width at half maximum (FWHM) and the divergence was 5 mrad. Fig. 1a and Fig. 1b show a scheme and photograph of the experimental setup, respectively.

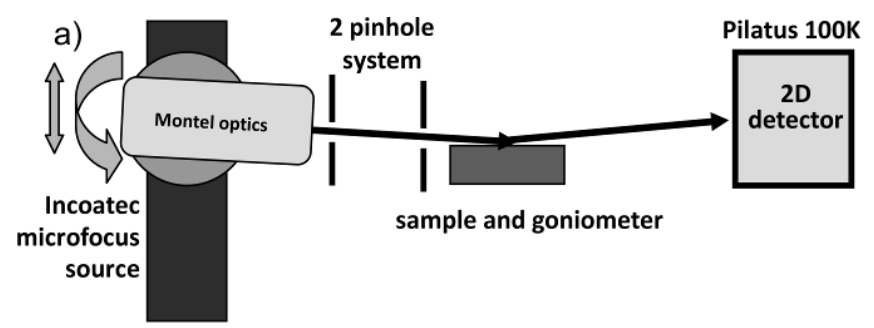

b)

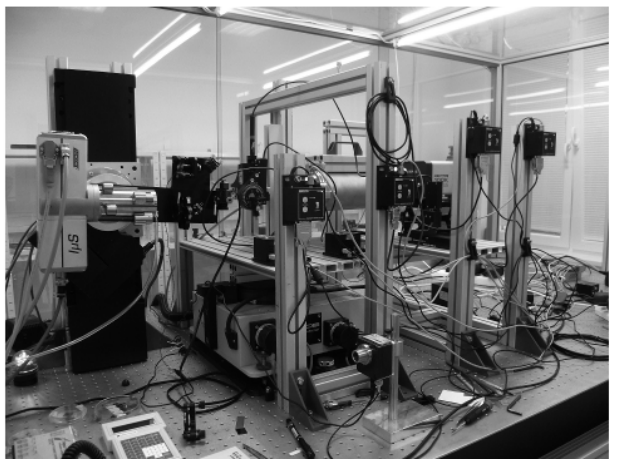

Fig.1. (a) The scheme and (b) photograph of the GISAXS apparatus.

The X-ray source is attached via a rotation stage to a vertical linear stage. This setup allows a precise adjustment of the X- 
ray beam and the sample surface. A collimation block consists of two pinholes. The first pinhole blocks the nonspecular scattering from the Montel optics. The second pinhole forms the X-ray beam. In our case, the second pinhole was a circular one with a diameter of $100 \mu \mathrm{m}$ drilled by a laser beam into a $0.25 \mathrm{~mm}$ thick tungsten foil. The distance between the two pinholes is $100 \mathrm{~mm}$. The measured divergence of the collimated X-ray beam is $0.8 \mathrm{mrad}$ that corresponds to theoretical resolution of $0.03 \mathrm{~nm}^{-1}$ in the reciprocal space. The sample is located at a compact rotation stage supported by a vertical linear stage. The distance between the second pinhole and the sample is $80 \mathrm{~mm}$. The scattered X-ray radiation is detected by a two dimensional semiconductor X-ray detector (Pilatus 100K, Dectris). The detector allows time-resolved measurements with the exposure time down to $5 \mathrm{~ms}$. The distance between the sample and detector was set to $600 \mathrm{~mm}$. The direct beam was stopped by a tungsten beamstop located in front of the detector.
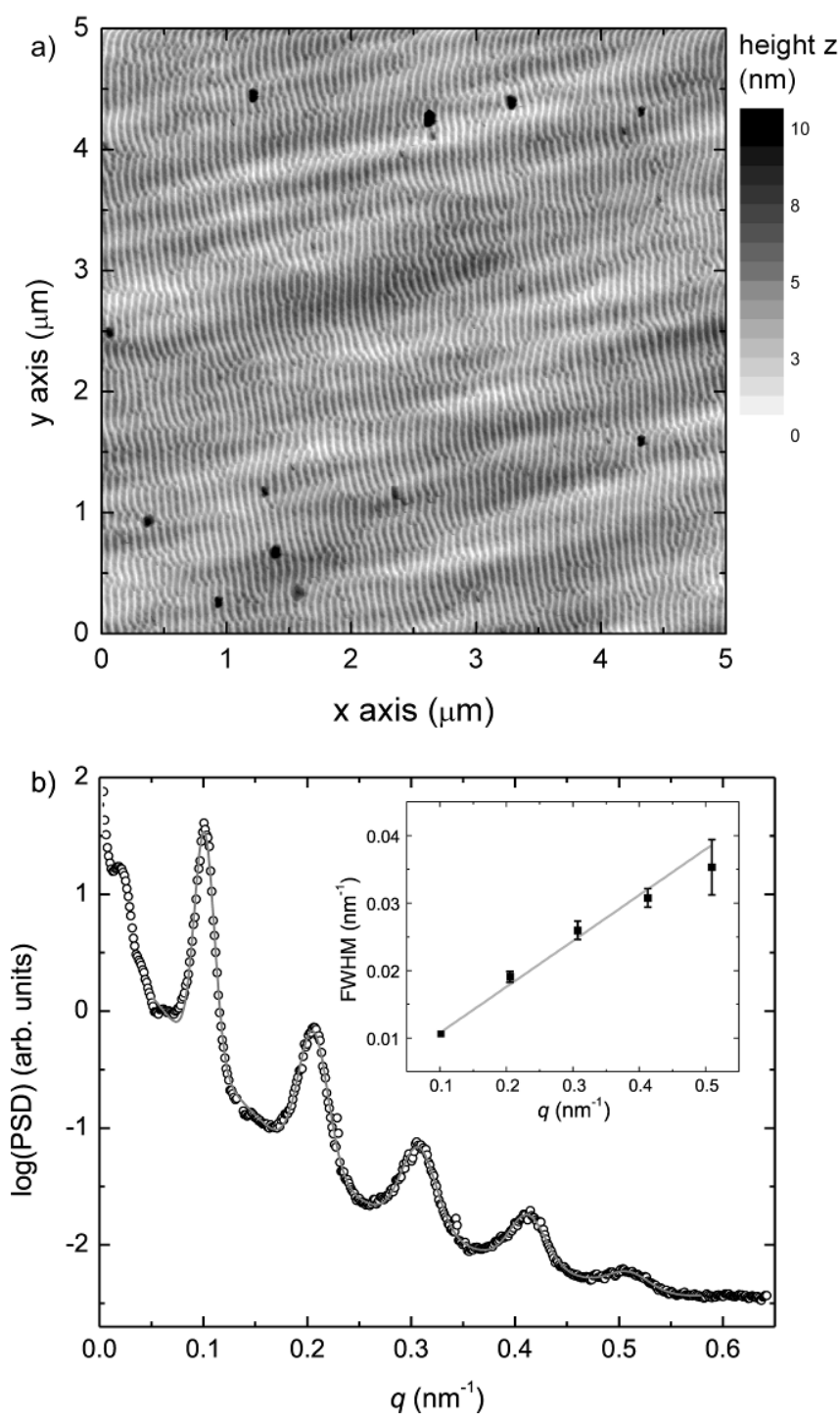

Fig.2. (a) AFM image of the surface. (b) The PSD function of the surface morphology. The inset shows the increasing broadening of the peak widths typical for the second-type of disorder.

\section{RESULTS \& DiSCUSSION}

The AFM image of the sample surface is shown in Fig. 2a. The scan area was $5 \times 5 \mu \mathrm{m}^{2}$ with a step size of $4.9 \mathrm{~nm}$. The AFM image of the ion-beam etched silicon substrate shows periodical ripples with typical amplitude of approx. $1 \mathrm{~nm}$. The rms (root mean square) roughness of the total scanned area is $1.3 \mathrm{~nm}$. The averaged power spectral density (PSD) function calculated over all scanned lines in horizontal direction is shown in Fig. 2b. A series of peaks observed in the PSD function, that are separated by a constant value $\Delta q$, suggests a regular periodicity with a period of $\Lambda=2 \pi / q$ in the real space [4]. The average peak spacing $\Delta q=0.102 \pm 0.001 \mathrm{~nm}^{-1}$ gives a mean ripple period $\Lambda=61.6 \pm 0.6 \mathrm{~nm}$. The broadening of the peaks in the PSD function indicates a gradual loss of the long-range order classified also as a second-type disorder [5]. In the first approximation, the widths (FWHM) of the peaks $\delta q$ vary as a linear function of the lateral frequency $q[6,7]$.

$$
\delta q=\delta q_{0}+\gamma q
$$

Here $\delta q_{0}=2 \pi / \xi$ where $\xi$ is the lateral correlation length of the ripples and $\gamma$ is the disorder parameter [2]. The inset of Fig. $2 b$ shows a linear fit of the experimental peak widths. Based on the fit parameters, the value of the lateral correlation length is $\xi=2.1 \pm 0.6 \mu \mathrm{m}$. The disorder parameter $\gamma=7.5 \pm 0.5 \%$.
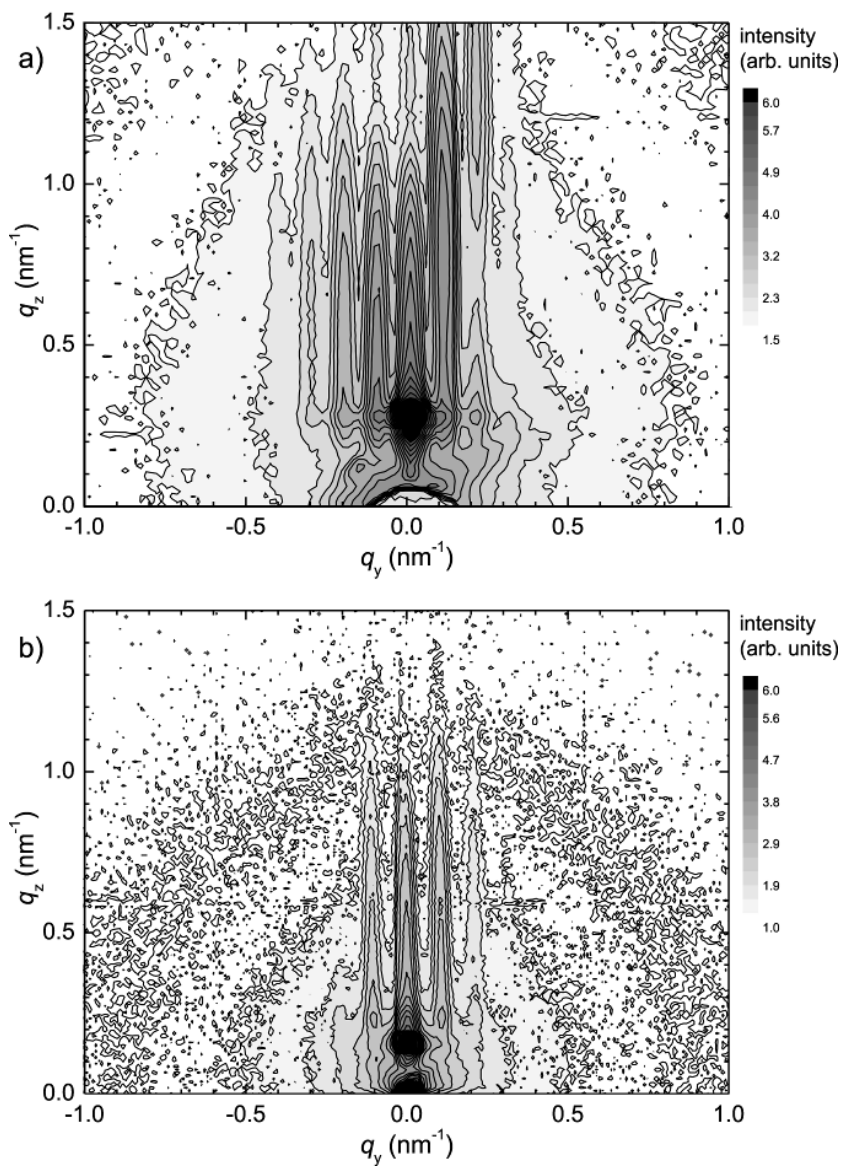

Fig.3. The GISAXS maps of ripple formations obtained by (a) $\mathrm{Cu}-\mathrm{K}_{\alpha}$ and (b) $\mathrm{Cr}-\mathrm{K}_{\alpha}$ radiation. 
The GISAXS technique is a non-contact measurement technique recording the reciprocal space image of the interface morphology [8]. Usually, the GISAXS studies have been confined to synchrotron beamlines because a collimated, monochromatic beam with a high brilliance is required. Here we present GISAXS reciprocal space maps obtained by a modular laboratory GISAXS system developed at the Institute of Physics SAS. Fig. 3a and Fig. $3 \mathrm{~b}$ show the reciprocal space maps measured by $\mathrm{Cu}-\mathrm{K}_{\alpha}$ and $\mathrm{Cr}-\mathrm{K}_{\alpha}$ lines, respectively. The collimation apertures were identical in both experimental setups. The intensity of the primary beam for the $\mathrm{Cu}-\mathrm{K}_{\alpha}$ line is $10 \mathrm{x}$ higher than that of the $\mathrm{Cr}-\mathrm{K}_{\alpha}$ line measured at the detector position. The reason is the higher power output of the $\mathrm{Cu}-\mathrm{K}_{\alpha} \mathrm{X}$-ray source and lower air absorption at this emission line. Therefore, the space map recorded with the $\mathrm{Cu}-\mathrm{K}_{\alpha}$ radiation has a higher dynamical range and more scattering maxima are visible.
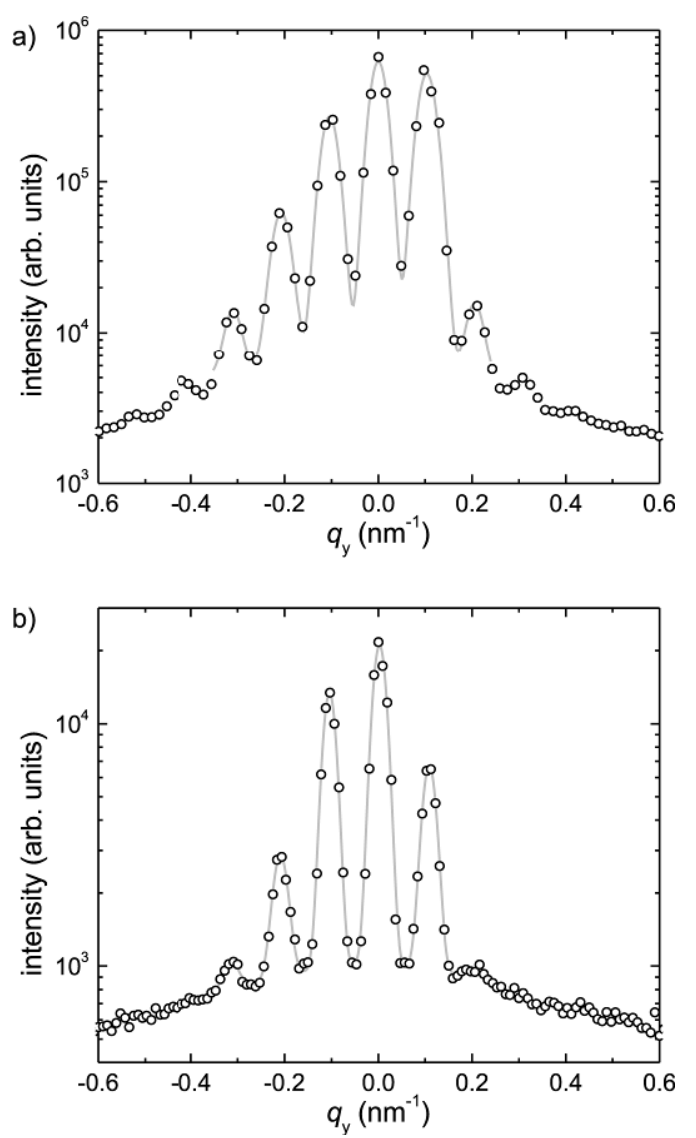

Fig.4. Integrated intensity profiles along $q_{\mathrm{y}}$ direction extracted from the reciprocal space maps recorded by (a) $\mathrm{Cu}-\mathrm{K}_{\alpha}$ and (b) $\mathrm{Cr}-\mathrm{K}_{\alpha} \mathrm{X}$-ray emission lines.

For the analysis we integrated the horizontal line cuts for $q_{z}$ value in the interval between $0.5 \mathrm{~nm}^{-1}$ and $1 \mathrm{~nm}^{-1}$. The integration results are shown in Fig. $4 \mathrm{a}$ and Fig. $4 \mathrm{~b}$ for $\mathrm{Cu}-\mathrm{K}_{\alpha}$ and $\mathrm{Cr}-\mathrm{K}_{\alpha}$ reciprocal space maps, respectively. The width of the scattering peaks is in both cases given by the instrumental resolution of the GISAXS apparatus. Assuming the large lateral correlation length of the periodic ripple structure (more than $2 \mu \mathrm{m}$ as calculated from the AFM data), we can use the width of the scattering peaks to determine the instrumental resolution of the GISAXS apparatus. For the $\mathrm{Cu}-\mathrm{K}_{\alpha}$ data (Fig. $4 \mathrm{a}$ ), the instrumental resolution based on the fitted width (FWHM) of the peaks is $0.042 \pm 0.001 \mathrm{~nm}^{-1}$ that corresponds to the maximum resolvable period of $150 \pm 4 \mathrm{~nm}$ in the real space. The same procedure for the $\mathrm{Cr}-\mathrm{K}_{\alpha}$ data (Fig. 4b) provides the instrumental resolution of $0.034 \pm 0.002 \mathrm{~nm}^{-1}$ that corresponds to $185 \pm 11 \mathrm{~nm}$ in real space. The instrumental resolution values obtained agree well with the calculated value of $0.03 \mathrm{~nm}^{-1}$ based on the geometrical considerations. The better instrumental resolution in the case of the $\mathrm{Cr}-\mathrm{K}_{\alpha}$ emission line is attributed to the $\mathrm{X}$-ray wavelength $1.5 \mathrm{x}$ larger than for the $\mathrm{Cu}-\mathrm{K}_{\alpha}$ emission line as all other experimental parameters remained unchanged. For a single surface the scattering intensity along the $q_{\mathrm{y}}$ direction is proportional to the PSD of the surface height function. This is valid within the Born approximation [9] and in the limit of low surface roughness [10]. The separation of the adjacent scattering peaks $\Delta q$ corresponds to the period $\Lambda=2 \pi / \Delta q$ of the surface structure. The values $\Delta q$ and $\Lambda$ obtained from fitting the experimental data by Gaussian function are summarized in Tab. 1 together with the data from the AFM analysis.

Table 1 Parameters of the surface obtained from GISAXS and AFM.

\begin{tabular}{|c|c|c|c|}
\hline & Cu- $\mathrm{K}_{\alpha}$ line & Cr- $_{\alpha}$ line & AFM \\
\hline$\Delta q\left(\mathrm{~nm}^{-1}\right)$ & $0.104 \pm 0.001$ & $0.106 \pm 0.001$ & $0.102 \pm 0.001$ \\
\hline$\Lambda(\mathrm{nm})$ & $60.4 \pm 0.6$ & $59.3 \pm 0.6$ & $61.6 \pm 0.6$ \\
\hline
\end{tabular}

The values of surface period $\Lambda$ provided by GISAXS technique at the two distinct X-ray emission lines coincide within the standard deviation limits. The slightly larger value of the period $\Lambda$ estimated by the AFM is due to the local character of the scanning probe technique which is restricted to a randomly selected inspection area of some $25 \mu \mathrm{m}^{2}$. On the contrary, the footprint of the probing X-ray beam on the sample surface is approximately $0.4 \mathrm{~cm}^{2}$ which guarantees a non-local averaged value of the surface period obtained. The advantage of the AFM technique over the GISAXS lies in the inherently larger interval for probing the lateral correlation length. The GISAXS X-ray wavelength is some 50x smaller than the nominal tip radius of the conventional AFM cantilever. A highly collimated X-ray beam is required to probe lateral correlation length at micrometer scale which is available at the $3^{\text {rd }}$ generation synchrotron beamlines [2].

\section{CONCLUSION}

In this contribution we presented an innovative laboratory GISAXS system capable of statistical characterization of surface morphology at the nanometer scale. Traditional scanning probe techniques are not suitable for the in-situ monitoring and control as they are local and working in the proximity of the inspected surface. On the contrary, the GISAXS is a non-local technique capable of giving fast statistical information on the surface morphology. The low scattering cross-section of the emission X-ray lines used 
allows the GISAXS measurement to be performed at atmospheric pressure without a significant deterioration of the data quality. We compared the AFM and GISAXS techniques using a silicon surface etched by ion-beam sputtering. The mean period of the ion-beam induced ripples at sample surface estimated by the GISAXS technique matches well the period obtained from the AFM measurements.

\section{ACKNOWLEDGMENT}

This publication is the result of the project implementation: Centre of Excellence for New Technologies in Electrical Engineering, ITMS code 26240120011, supported by the Research \& Development Operational Programme funded by the ERDF.

\section{REFERENCES}

[1] Ziberi, B., Frost, F., Hoche, T., Rauschenbach, B. (2005). Ripple pattern formation on silicon surfaces by low-energy ion-beam erosion: Experiment and theory. Phys. Rev. B 72, 235310.

[2] Carbone, D., Biermanns, A., Ziberi, B., Frost, F., Plantevin, O., Pietsch, U., Metzger, T.H. (2009). Ioninduced nanopatterns on semiconductor surfaces investigated by grazing incidence $\mathrm{x}$-ray scattering techniques. J. Phys.-Condens. Mat. 21, 224007.

[3] Ziberi, B., Cornejo, M., Frost, F., Rauschenbach, B. (2009). Highly ordered nanopatterns on $\mathrm{Ge}$ and $\mathrm{Si}$ surfaces by ion beam sputtering. J. Phys.-Condens. Mat. 21, 224003.
[4] Bennett, J.M., Mattsson, L. (1989). Introduction to Surface Roughness and Scattering. Washington, D.C.: Optical Society of America.

[5] Guinier, A. (1963). X-ray Diffraction in Crystals, Imperfect Crystals, and Amorphous Bodies. San Francisco: W.H. Freeman.

[6] [6] Dullens, R.P.A., Petukhov, A.V. (2007). Secondtype disorder in colloidal crystals. Epl.-Europhys. Lett. 77, 58003.

[7] Petukhov, A.V., Thijssen, J.H.J., 't Hart, D.C., Imhof, A., van Blaaderen, A., Dolbnya, I.P., Snigirev, A., Moussaid, A., Snigireva, I. (2006). Microradian X-ray diffraction in colloidal photonic crystals. J. Appl. Crystallogr. 39, 137-44.

[8] Renaud, G., Lazzari, R., Leroy, F. (2009). Probing surface and interface morphology with Grazing Incidence Small Angle X-Ray Scattering. Surf. Sci. Rep. 64, 255-380.

[9] Born, M., Wolf, E. (1999). Principles of Optics : Electromagnetic Theory of Propagation, Interference and Diffraction of Light. Cambridge; New York: Cambridge University Press.

[10] Daillant, J., Gibaud, A. (2009). X-Ray and Neutron Reflectivity : Principles and Applications. Berlin; Heidelberg: Springer. 